\title{
BMJ Open Increasing prevalence of NAFLD/NASH among children, adolescents and young adults from 1990 to 2017: a population- based observational study
}

\author{
Xin Zhang (D) , ${ }^{1}$ Mingshan Wu, ${ }^{1}$ Zhenqiu Liu, ${ }^{2,3,4}$ Huangbo Yuan, ${ }^{1}$ Xuefu Wu, ${ }^{1}$ \\ Tingting Shi, ${ }^{1}$ Xingdong Chen, ${ }^{2,3,4}$ Tiejun Zhang ${ }^{1,3}$
}

To cite: Zhang X, Wu M, Liu Z, et al. Increasing prevalence of NAFLD/NASH among children, adolescents and young adults from 1990 to 2017: a population-based observational study. BMJ Open 2021;11:e042843. doi:10.1136/ bmjopen-2020-042843

- Prepublication history for this paper is available online. To view these files, please visit the journal online ()

$\mathrm{XZ}$ and MW contributed equally.

Received 20 July 2020

Revised 02 March 2021

Accepted 17 April 2021

Check for updates

(C) Author(s) (or their employer(s)) 2021. Re-use permitted under CC BY-NC. No commercial re-use. See rights and permissions. Published by BMJ.

${ }^{1}$ Department of Epidemiology, School of Public Health, Fudan University, Shanghai, China

${ }^{2}$ State Key Laboratory of Genetic Engineering and Collaborative Innovation Center for Genetics and Development, School of Life Sciences, Fudan University, Shanghai, China

${ }^{3}$ Fudan University Taizhou Institute of Health Sciences, Taizhou, China

${ }^{4}$ Human Phenome Institute, Fudan University, Shanghai, China

Correspondence to PhD Tiejun Zhang; tjzhang@shmu.edu.cn

\section{ABSTRACT}

Objective To describe the prevalence and variations of non-alcoholic fatty liver disease/non-alcoholic steatohepatitis (NAFLD/NASH) among children and adolescents (CADs) and young adults (YADs).

Design A population-based observational study. Setting Annual cases and prevalence of NAFLD/NASH from 1990 to 2017, by sex, region and country were collected from the Global Burden of Disease database. Main outcome measures The estimated annual percentage change, which was calculated by a regression line, was used to quantify the temporal trends in NAFLD/ NASH burden among young people at the global, regional and national levels.

Results Globally, NAFLD/NASH incidence increased from 19.34 million in 1990 to 29.49 million in 2017 among CADs, with an annual increase of $1.35 \%$. Additionally, in YADs, the number of cases and NAFLD/NASH prevalence significantly increased during this period, independent of sex and region. The greatest NAFLD/NASH increase was in North Africa and the Middle East. Almost all countries showed an increasing trend from 1990 to 2017, with the most pronounced increase observed in the developed regions.

Conclusions The epidemiology of NAFLD/NASH in young people has changed considerably over the last three decades. Both the prevalence and number of cases have increased irrespective of sex, age and region. This phenomenon can result in a predictable increase in chronic liver disease burden in the near future. Understanding the prevalence of NAFLD/NASH and its variations is of paramount importance to develop strategies to implement public health policy.

\section{INTRODUCTION}

Over the past three decades, lifestyle transformation has radically changed health priorities worldwide, owing to the growing incidence of non-communicable disease. ${ }^{1}$ The new epidemic of chronic liver disease (CLD) is related to the increasing prevalence of non-alcoholic fatty liver disease (NAFLD) that parallels the worldwide increase in obesity. ${ }^{2}$ According to a meta-analytic, the
Strengths and limitations of this study

- The specific situation of non-alcoholic fatty liver disease/non-alcoholic steatohepatitis (NAFLD/NASH) among children and adolescents and young adults (YADs) requires further exploration. Our study can provide evidence of the global, regional and national prevalence and its variations in NAFLD/NASH among the young population to address this gap.

- Our study also uses the estimated annual percentage change to quantify the secular trends in the prevalence of NAFLD/NASH.

- The analyses were based on data from the Global Burden of Disease dataset, and the miscoding and under-reporting of NAFLD/NASH because of a lack of medical information can cause bias in the estimations.

- Because of the population-based design, individuallevel data were unavailable; therefore, we could not estimate the contributions of risk factors to NAFLD/ NASH over time.

- The diagnosis methods of every country were not known, which may have influenced the prevalence estimation. Although most countries use ultrasound or other imaging diagnostics, different diagnostic methods can lead to different prevalence.

global prevalence of NAFLD is $25.24 \%$ and has a high prevalence in all continents, with the highest reported in the Middle East $(31.78 \%)$ and South America (30.45\%), while the lowest in Africa $(13.48 \%){ }^{3}$ A smaller subgroup of these patients can develop nonalcoholic steatohepatitis (NASH), which has been considered as the most common aetiologies for hepatocellular carcinoma (HCC) and indications for liver transplantation in the USA. ${ }^{4}$

With obesity shifting towards an early onset in life ${ }^{5-7}$ NAFLD/NASH prevalence began to increase in the younger populations. In the Western countries, 3\%-10\% of children are estimated to be affected by NAFLD. ${ }^{8}$ 
However, given the difficulty in its diagnosis in children, the rate is underestimated, ${ }^{9}$ and in contrast with that in adults, the disease in children has been ignored by healthcare providers. The rate among people aged 18-35 years in America is approximately $24 \%$, which has also been underestimated. Compared with adults, they do not have adequate health insurance coverage and a delay in diagnosis attributed to their sense of invulnerability and lack of awareness among physicians. ${ }^{10}$ In addition, evidence to inform management practices and policies for children, adolescents and young adults (YADs) had major gaps. According to a recent study, NAFLD/NASH caused by overweight in late adolescence is a significant predictor of severe liver disease, including HCC later in life. ${ }^{11}$ The specific situation of NAFLD/NASH requires further exploration. Although some studies have reported the prevalence of NAFLD/NASH among children, most of them have been regionally limited and some regions have not been systematically investigated. ${ }^{10}$ To address this gap, this study aimed to provide evidence of the global, regional and national prevalence and its variations in NAFLD/NASH among the young population, which should be considered by health policies to secure a better future for the coming generations of children.

\section{MATERIALS AND METHODS \\ Data collection}

Annual cases and prevalence of NAFLD/NASH from 1990 to 2017 by sex, region and country were collected from the Global Health Data Exchange (GHDx) query tool (http://ghdx.healthdata.org/gbd-results-tool). ${ }^{12}$ Data from 195 countries and territories are available. These countries and territories were then categorised into five regions based on the Socio-Demographic Index (SDI), including low, low middle, middle, high middle and high. Moreover, the world is divided into 21 geographical regions, such as East Asia. The general methods for the Global Burden of Disease (GBD) 2016 and the methods for estimating disease burden have been detailed in previous studies. ${ }^{13} 14$ Data of people aged 39 years and younger were retrieved. People aged 0-19 years and 20-39 years were identified as children and adolescents (CADs) and YADs, respectively. NAFLD is broad category for a range of liver conditions affecting people who drink little to no alcohol and is identified by the ICD10 code K76.0. NAFLD prevalence was estimated by a systematic literature review of PubMed and then modelled using DisMod-MR 2.1 models. ${ }^{13}$

\section{Patient and public involvement}

The data for this study were obtained from the GHDx query tool. No clinical information was extracted for this study.

\section{Statistical analysis}

The estimated annual percentage change (EAPC) was used to quantify the prevalence trends of NAFLD/NASH, stratified by sex, region, nation and age, from 1990 to 2017. The EAPC was calculated by a regression line, which was fitted to the natural logarithm of the rates, that is, $y=\alpha+\beta x+\varepsilon$, where $y=\ln ($ rate $)$ and $x=$ calendaryear. The rate was deemed to increase if the EAPC estimation and the lower limit of its $95 \%$ CI were both $>0$. By contrast, the rate decreased if the EAPC estimation and the upper limit of its $95 \%$ CI were both $<0$. Otherwise, the rate was deemed stable over time. All statistical analyses were performed using R (V.3.5.1, R Core Team, Vienna, Austria). Statistical significance was set at $\mathrm{p}<0.05$.

\section{RESULTS \\ Prevalence of NAFLD/NASH and its variations among CADs and YADs at the global level}

Globally, the prevalence of NAFLD/NASH increased from 19.34 million in 1990 to 29.49 million in 2017 among CADs, with an annual increase of $1.35 \%$ during this period. The increase was more pronounced in boys than in the girls (boys: $\mathrm{EAPC}=1.36,95 \% \mathrm{CI} 1.18$ to 1.55 ; girls: $\mathrm{EAPC}=1.31,95 \% \mathrm{CI} 1.12$ to 1.49 ) and approximately $2 / 3$ of the total NAFLD/NASH cases occurred in boys in 2017 (table 1). Both the number of cases and prevalence of NAFLD/NASH were higher in regions with high SDIs than in regions with relatively low SDIs (table 1, figure 1A). All SDI regions had a significant increase in NAFLD/NASH prevalence among CADs, with the greatest increase observed in the high-middle SDI regions (table 1 ).

Similarly, the number of cases and prevalence of NAFLD/NASH among YADs also showed a significant increase from 1990 to 2017 independent of the sex and region (table 1, figure 1B). And the number of cases of NAFLD/NASH among YADs was higher than that in CADs (152.85 million in 1990; 284.32 million in 2017). The prevalence of all five SDI regions increased almost linearly, with the high-middle SDI region having the highest prevalence $(15.04 \%)$.

\section{Prevalence of NAFLD/NASH and its variations among CADs and YADs at the regional level}

All GBD regions demonstrated an increasing trend in NAFLD/NASH prevalence among the CAD patients. In 2017, a relatively higher prevalence was observed in North Africa and the Middle East (2.05\%), followed by Central Latin America (1.88\%), High-income North America (1.64\%), Oceania (1.57\%) and Andean Latin America $(1.56 \%)$. The lowest regions were Eastern Sub-Saharan Africa, Central Sub-Saharan Africa and Western Sub-Saharan Africa $(0.62 \%, 0.66 \%$ and $0.70 \%$, respectively) (figure 2A). The prevalence in YADs was approximately 10-fold of that in CADs, while the trend of NAFLD/NASH prevalence was similar to that in CADs. In 2017, a relatively high prevalence of NAFLD/NASH in YADs was observed in North Africa and the Middle East (22.18\%), followed by that in Oceania (17.14\%), Central Latin America (16.20\%) and Southeast Asia (15.41\%). 
Table 1 The cases, prevalence and EAPC of NAFLD/NASH among CADs and YADs in 1990 and 2017 by sex and SDI region NAFLD/NASH

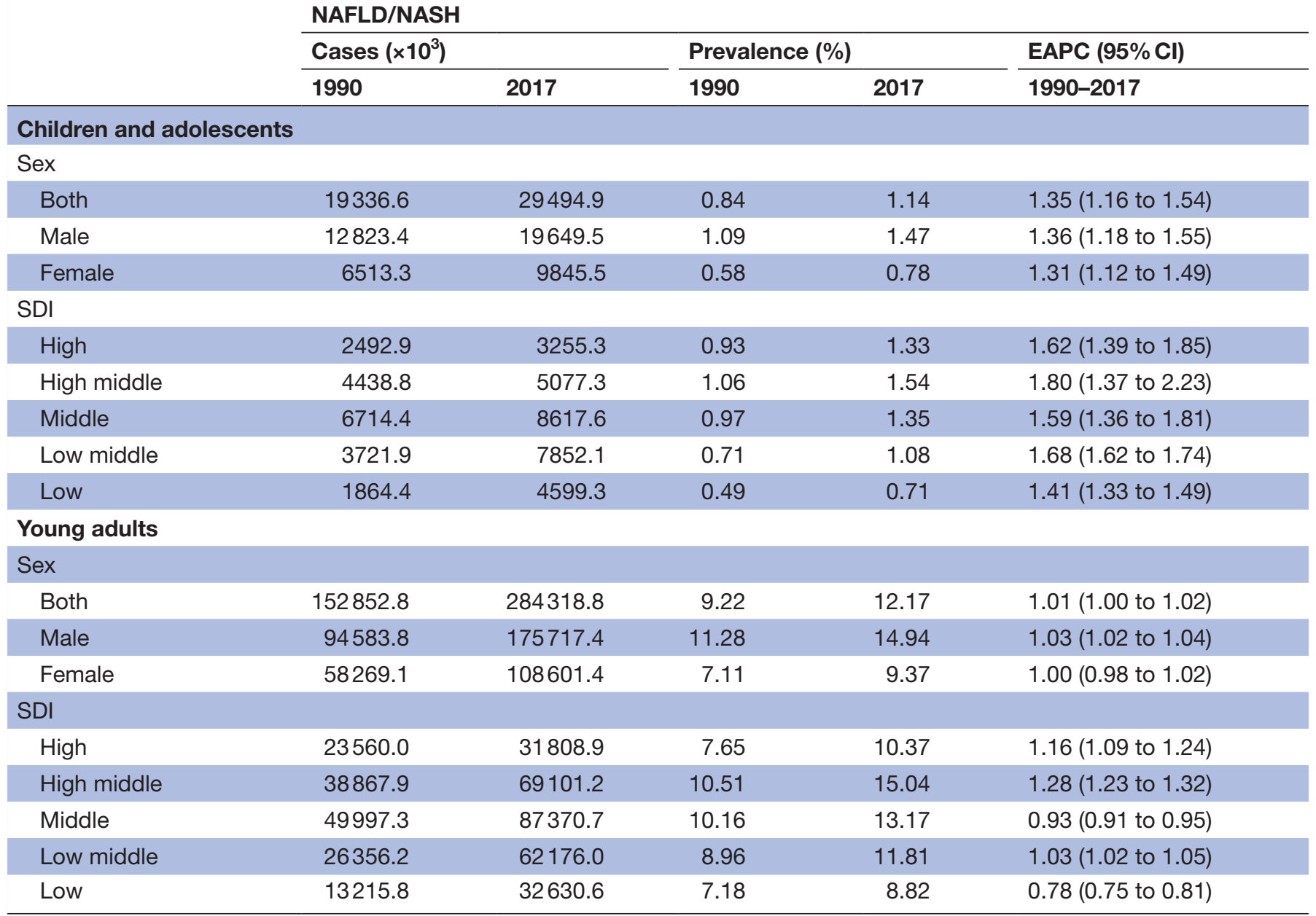

CADs, children and adolescents; EAPC, estimated annual percentage change; SDI, Socio-Demographic Index; YADs, young adults.

The lowest prevalence were observed in the Eastern Sub-Saharan Africa, Central Sub-Saharan Africa and Central Europe $(7.88 \%, 8.46 \%$, and $8.10 \%$, respectively) (figure 2B).

The greatest increase in CADs was observed in highincome North America (EAPC=2.23, 95\% CI 1.92 to 2.54), and the lowest increase occurred in Eastern Europe $(\mathrm{EAPC}=0.64,95 \% \mathrm{CI}-0.08$ to 1.37 ) (figure $3 \mathrm{~A}$ ). However, in YADs, the greatest increase was observed in Central Latin America (EAPC=1.30, 95\% CI 1.26 to 1.34 ) and a minimum increase was observed in Central Sub-Saharan Africa (EAPC=0.56, 95\% CI 0.49 to 0.63 ) (figure 3B).

\section{Prevalence of NAFLD/NASH and its variations among CADs} and YADs at the national level

Among the CADs in 195 countries, the highest prevalence was found in American Samoa (3.34\%) and Saudi Arabia $(3.13 \%)$, and the lowest prevalence was observed in Greenland $(0.15 \%)$ and Andorra (0.16\%). Between 1990 and 2017, only three countries (EAPC: the Czech Republic: -0.44 to $0.80,-0.09$; Bulgaria: -0.32 to 0.62 , -0.02 ; Somalia: -0.15 to $0.47,0.17$ ) showed a significant decrease during this period. More than $95 \%$ of the countries (188/195) experienced a significant increase in NAFLD/NASH prevalence, with the most pronounced increase observed in the Maldives (EAPC $=3.55,95 \%$ CI 3.06 to 4.05 ) (figure 4 ).

Compared with CADs, the highest prevalence of NAFLD/NASH among YADs was observed in countries located in the Middle East, such as Qatar (29.44\%) and Kuwait $(27.09 \%)$. The lowest prevalence was observed in Greenland (1.28\%) similar to that of the CADs. Only one country (Bulgaria: $\mathrm{EAPC}=0.15,95 \% \mathrm{CI}-0.01$ to 0.31 ) remained stable in NAFLD/NASH prevalence, whereas the remaining 194 countries or territories showed a significant increasing trend from 1990 to 2017. The greatest increase was observed in Oman $(\mathrm{EAPC}=2.20$, $95 \%$ CI 2.00 to 2.40), followed by that in Equatorial Guinea (EAPC $=1.86,95 \%$ CI 1.73 to 2.00 ) and Nicaragua (EAPC $=1.71,95 \%$ CI 1.61 to 1.80$)$. Generally, countries with a high prevalence also have a high EAPC, while some are different, such as YADs in Finland, the prevalence was low $(8.20 \%)$, while the EAPC was high $(\mathrm{EAPC}=1.70$ $95 \%$ CI 1.55 to 1.84 ) (figure 4 ). 

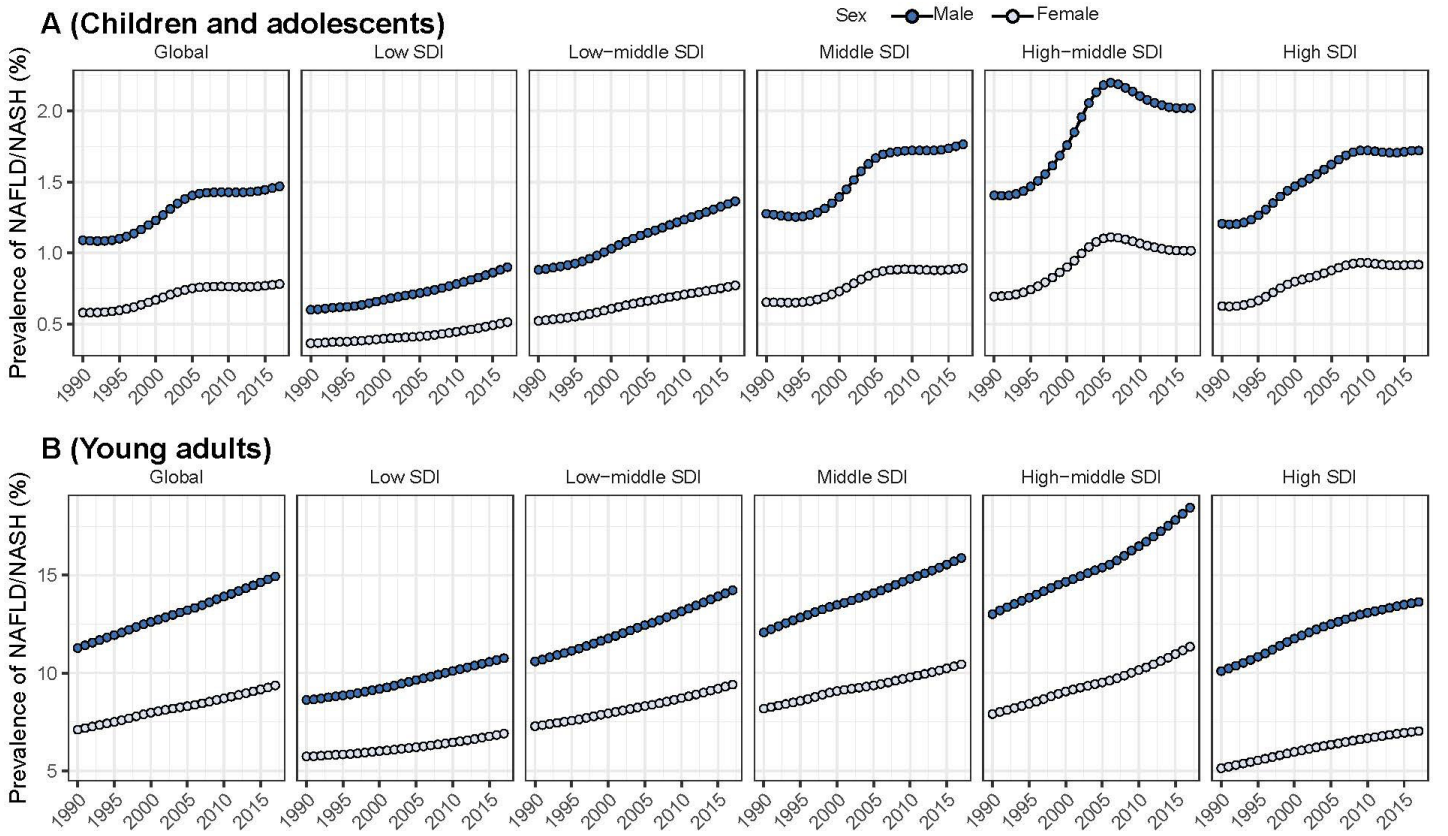

Figure 1 The temporal trends of the prevalence of non-alcoholic fatty liver disease/non-alcoholic steatohepatitis (NAFLD/ NASH) (\%) among children and adolescents and young adults from 1990 to 2017 by sex and Socio-Demographic Index (SDI) region.

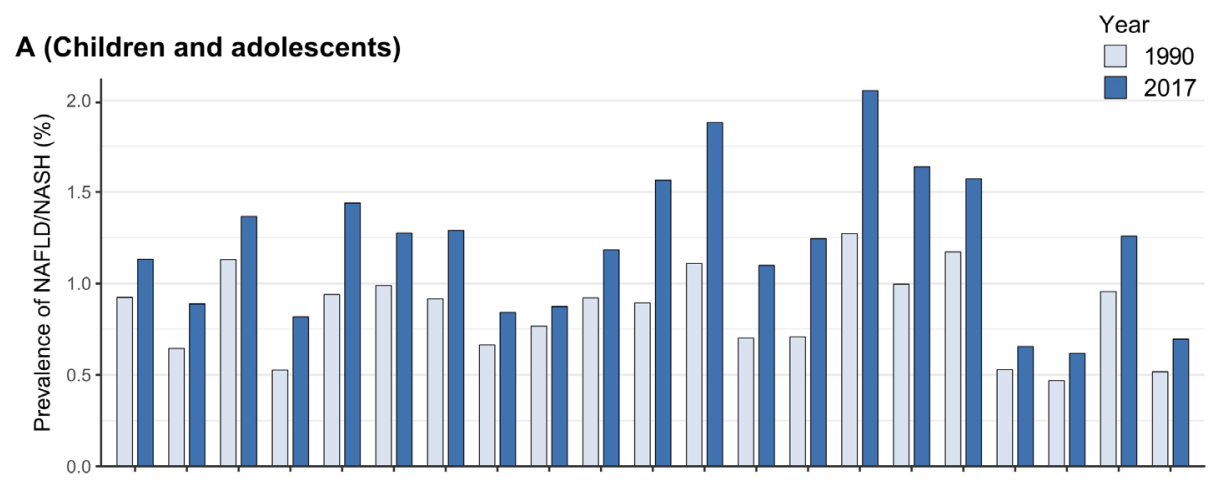

B (Young adults)

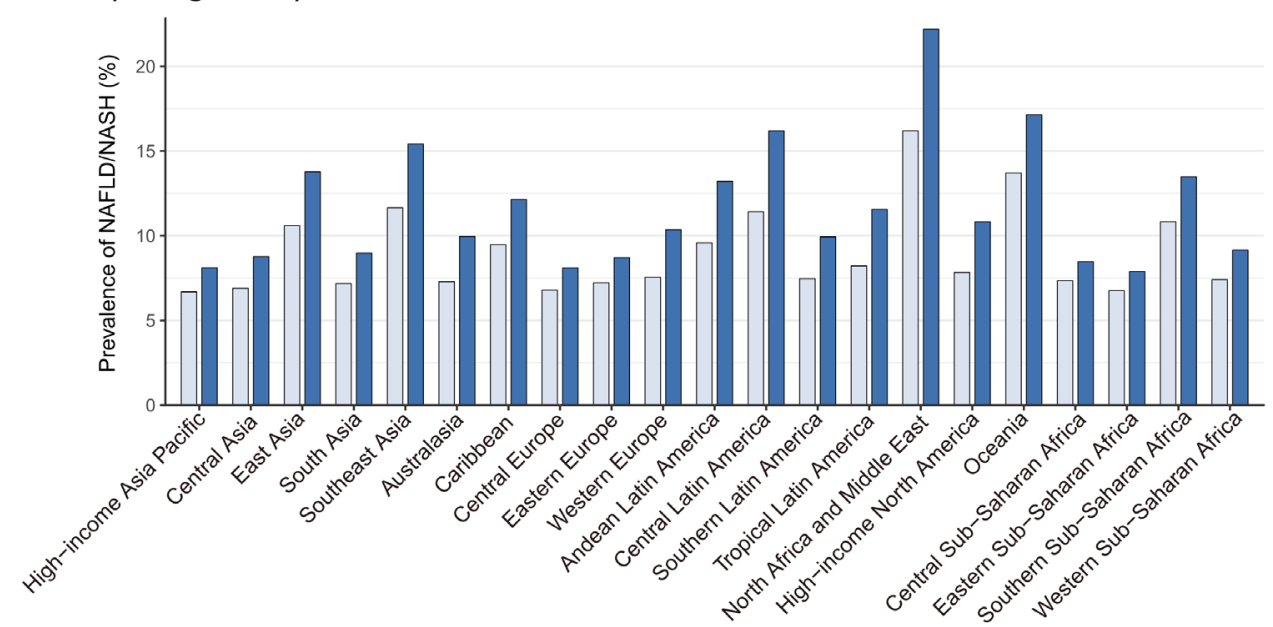

Figure 2 The prevalence of non-alcoholic fatty liver disease/non-alcoholic steatohepatitis (NAFLD/NASH) (\%) among children and adolescents and young adults in 1990 and 2017 at the global burden of disease-region level. 
A (Children and adolescents)

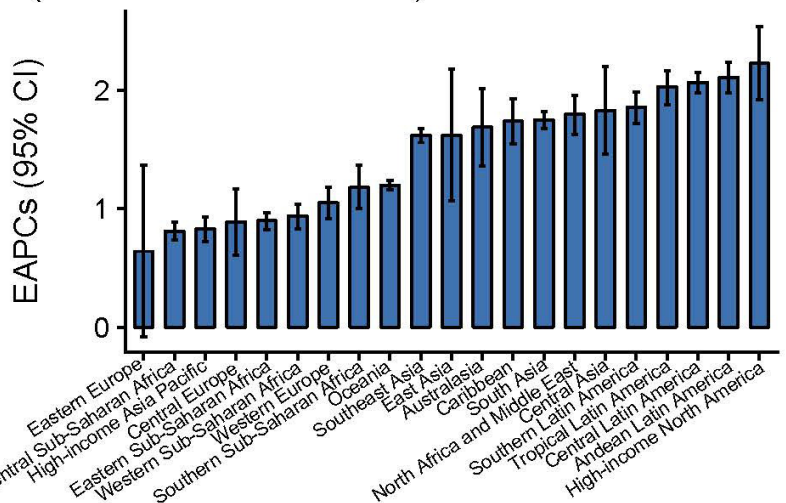

B (Young adults)

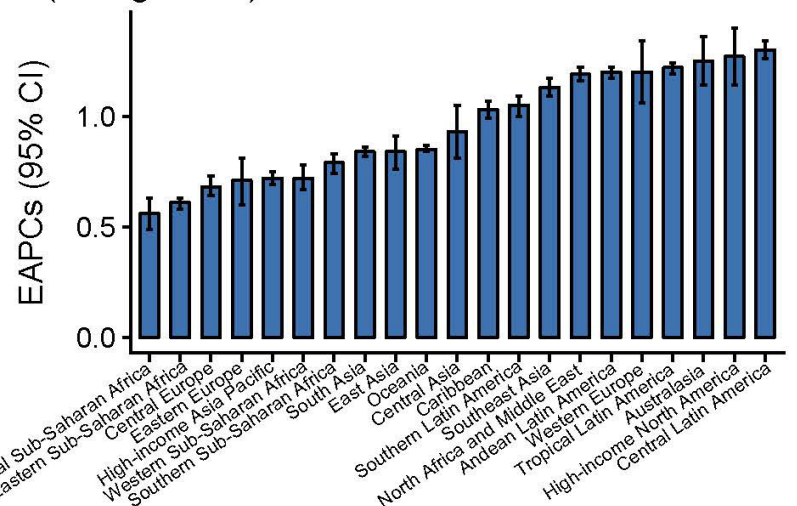

Figure 3 The estimated annual percentage changes (EAPCs) in the prevalence of non-Icoholic fatty liver disease/non-alcoholic steatohepatitis among children and adolescents and young adults at the global burden of disease-regional level between 1990 and 2017. The error bar denotes the $95 \% \mathrm{Cl}$ for EAPC.

\section{DISCUSSION}

Over the past 30 years, NAFLD/NASH has shifted from a feature that could not be distinguish from alcoholic liver disease to the biggest causative factor of CLD worldwide, especially among CADs and YADs. ${ }^{15}$ With further modifications of contemporary lifestyle, ${ }^{16}$ NAFLD/NASH still has an increasing trend and will be predictably aggravated as the number of younger people with NAFLD will increase, which has received little attention. In this study, we analysed the prevalence and variations in NAFLD/ NASH prevalence among CADs and YADs at the global, regional and national levels. Generally, the number of cases and prevalence of NAFLD/NASH among these two populations both showed a significant increase from 1990 to 2017, independent of sex and region. The prevalence of NAFLD/NASH was found to be relatively high in North Africa and the Middle East, and the prevalence of NAFLD/NASH increased in these two populations during the same period.

Obesity is the main risk factor for NAFLD/NASH ${ }^{16-18}$; however, type 2 diabetes mellitus, ${ }^{19}$ alcohol intake, ${ }^{20}$ an unhealthy lifestyle such as skipping meals, higher intake of fast food ${ }^{21}$ and physical inactivity ${ }^{22}$ can also affect the disease. Sex and ethnicity are predisposing factors as well. ${ }^{23}$ The interplay between these factors can lead to disease progression, which can result in cirrhosis, liver failure and HCC at a younger age than that was commonly recognised. ${ }^{10}$ In our study, we found that all SDI regions showed an increase in NAFLD/NASH, with the greatest increase observed in the high-middle SDI region. The main cause of this situation was increase in obesity. According to the latest results, between 1975 and 2016 , the prevalence of overweight increased from $5 \%$ to $27 \%$ in boys and $6 \%$ to $24 \%$ in girls. ${ }^{5} \mathrm{~A}$ twofold increase
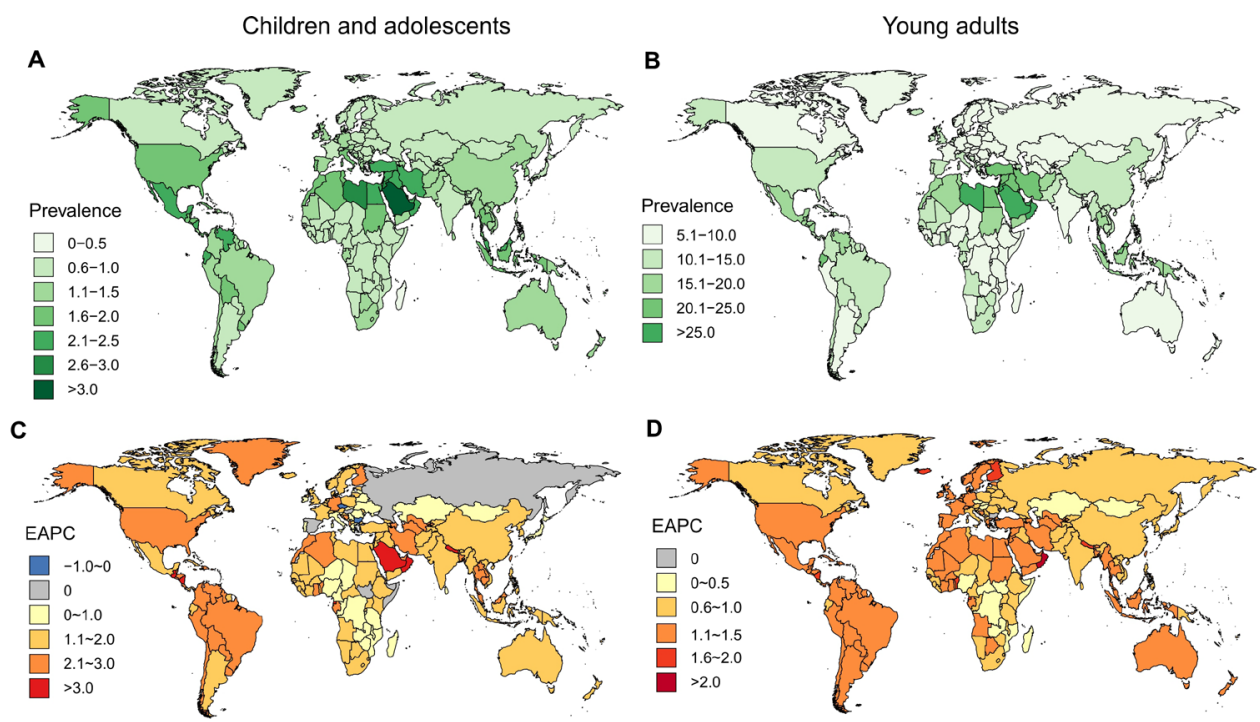

Figure 4 The prevalence and estimated annual percentage changes (EAPCs) of non-alcoholic fatty liver disease/non-alcoholic steatohepatitis (NAFLD/NASH) (\%) among children and adolescents (CADs) and young adults (YADs) in 2017 at the national level. (A) The prevalence of NAFLD/NASH among CADs in 2017 at the national level. (B) The prevalence of NAFLD/NASH among YADs in 2017 at the national level. (C) The EAPCs of NAFLD/NASH (\%) among CADs in 2017 at the national level. (D) The EAPCs of NAFLD/NASH (\%) among YADs in 2017 at the national level. 
in the prevalence was also found in people aged 20 years and older who were overweight. As a consequence of the obesity pandemic, NAFLD, especially the histological phenotype of NASH, has become one of the major causes of CLDs and will probably emerge as the leading cause of end-stage liver disease in the near future. ${ }^{1}$ The trend of the high-middle SDI region may be interpreted as a change in the prevalence of NAFLD/NASH in China. As the most populous country in the high-middle SDI region, prevalence in China rapidly increased from 1990 to 2005 and a relatively slow increase from 2005 to 2017. Generally, the number of cases increases with growth according to the SDI. Surprisingly, the prevalence of the high SDI region was relatively low in five regions. This may be due to the good medical measures and high health consciousness in this region, although the exact reasons need further investigation.

The sex factor among CADs worldwide is the same as YADs, with a higher prevalence in males. This may be explained by the different distributions of body fat in males and females patients. Compared with female patients, male patients tend to deposit more visceral adipose tissue in the abdominal region, a difference that becomes more marked in young adulthood. ${ }^{25-27}$ In a cross-sectional analysis, visceral fat was found to be an independent predictor of the degree of necroinflammation and stage of fibrosis in NAFLD. ${ }^{25}$ The prevalence of NAFLD/NASH among YADs is almost tenfold higher among CADs, indicating that the situation of the disease is more serious in YADs. The EAPC among CADs is higher than that among YADs, suggesting that we cannot overlook the disease in CADs. This phenomenon may be related to differences in sex hormones and insulin resistance among CADs and YADs,${ }^{28}$ which may be one of the causes of NAFLD in adolescents.

An increasing temporal trend of NAFLD/NASH has been detected, but high heterogeneity exists. For example, regarding CADs, the prevalence and EAPC are both high in high-income North America, Central Latin, America Andean Latin America and North Africa, suggesting a severe disease burden in these areas, which will still be heavy in the future. In YADs, Central Latin America has both a high prevalence and EAPC. In addition to obesity, the high prevalence of NAFLD in America is affected by genetic factors. Many studies have already suggested that Hispanic heritage with a polymorphism in the gene encoding a patatin-like phospholipase domain containing three genes has a higher prevalence than others. ${ }^{1529-31}$ Therefore, special attention must be given to these areas.

Among children, countries with relatively high prevalence and EAPC, such as Saudi Arabia and Qatar, need to take corresponding measures to control the disease. Countries with low prevalence but a high EAPC, such as Finland, should not be neglected as they are likely to develop a more severe situation in the future. Understanding the prevalence at the national level can help governments implement targeted measures.
Our study had some limitations. First, the analyses were based on data from the GBD dataset, and the miscoding and underreporting of NAFLD/NASH because of a lack of medical information support can cause bias in the estimations. Second, because of the population-based design, individual-level data were unavailable; therefore, we could not estimate the contributions of risk factors to NAFLD/ NASH over time. Finally, the diagnosis methods of every country were not known, which may have influenced the prevalence estimation. Although most countries use ultrasound or other imaging diagnostics, different diagnostic methods may lead to different prevalence. ${ }^{32}$

In summary, the epidemiology of NAFLD/NASH among young people has changed significantly over the last three decades. Although an increasing temporal trend exists worldwide, almost all countries show an increasing prevalence. Given the alarming increase in obesity among young people worldwide, the prevalence of NAFLD/NASH is anticipated to further increase in the near future. Understanding the prevalence of NAFLD/ $\mathrm{NASH}$ and its variations is of paramount importance to develop strategies to implement public health policy and deal with this important CLD.

Acknowledgements We appreciate the work by Global Burden of Disease Study 2017 collaborators. This work was supported by the National Key Research and Development Program of China (grant number: 2017YFC0907000, 2017YFC0907500, 2017YFC211700); the National Natural Science Foundation of China (grant numbers: 81772170, 81502870); the key basic research grants from the Science and Technology Commission of Shanghai Municipality (grant number: 16JC1400500); the International S\&T Cooperation ProgramME of China (grant number: 2015DFE32790) and Shanghai Municipal Science and Technology Major Project (2017SHZDZX01).

Contributors XZ, MW and TS were responsible for data collection. XW extracted the data and conducted the analysis with input from all authors. ZL and HY cleaned the data and analysed the data. XZ and MW conducted the initial literature searches and completed the initial drafts of the manuscript. XC and TZ reviewed the statistical method. All authors read and approved the final manuscript.

Funding This study was supported by National Natural Science Foundation of China (81772170) and by National Key Research and Development Program of China (No. 2017YFC0211700).

Map disclaimer The depiction of boundaries on the map(s) in this article does not imply the expression of any opinion whatsoever on the part of BMJ (or any member of its group) concerning the legal status of any country, territory, jurisdiction or area or of its authorities. The map(s) are provided without any warranty of any kind, either express or implied.

\section{Competing interests None declared.}

Patient and public involvement Patients and/or the public were not involved in the design, or conduct, or reporting, or dissemination plans of this research.

\section{Patient consent for publication Not required.}

Provenance and peer review Not commissioned; externally peer reviewed.

Data availability statement All data relevant to the study are included in the article or uploaded as online supplemental information. No additional data available.

Open access This is an open access article distributed in accordance with the Creative Commons Attribution Non Commercial (CC BY-NC 4.0) license, which permits others to distribute, remix, adapt, build upon this work non-commercially, and license their derivative works on different terms, provided the original work is properly cited, appropriate credit is given, any changes made indicated, and the use is non-commercial. See: http://creativecommons.org/licenses/by-nc/4.0/.

ORCID iD

Xin Zhang http://orcid.org/0000-0002-4814-6207 


\section{REFERENCES}

1 Younossi Z, Anstee QM, Marietti M, et al. Global burden of NAFLD and NASH: trends, predictions, risk factors and prevention. Nat Rev Gastroenterol Hepatol 2018;15:11-20.

2 Ayonrinde OT, Olynyk JK, Marsh JA, et al. Childhood adiposity trajectories and risk of nonalcoholic fatty liver disease in adolescents. J Gastroenterol Hepatol 2015;30:163-71.

3 Younossi ZM, Koenig AB, Abdelatif D, et al. Global epidemiology of nonalcoholic fatty liver disease-Meta-analytic assessment of prevalence, incidence, and outcomes. Hepatology 2016;64:73-84.

4 Anstee QM, Targher G, Day CP. Progression of NAFLD to diabetes mellitus, cardiovascular disease or cirrhosis. Nat Rev Gastroenterol Hepatol 2013;10:330-44.

5 Sung $\mathrm{H}$, Siegel RL, Torre LA, et al. Global patterns in excess body weight and the associated cancer burden. CA Cancer J Clin 2019;69:88-112.

6 Swinburn BA, Kraak VI, Allender S, et al. The global syndemic of obesity, undernutrition, and climate change: the Lancet Commission report. Lancet 2019;393:791-846.

7 NCD Risk Factor Collaboration (NCD-RisC). Worldwide trends in body-mass index, underweight, overweight, and obesity from 1975 to 2016: a pooled analysis of 2416 population-based measurement studies in 128.9 million children, adolescents, and adults. Lancet 2017;390:2627-42.

8 Alisi A, Feldstein AE, Villani A, et al. Pediatric nonalcoholic fatty liver disease: a multidisciplinary approach. Nat Rev Gastroenterol Hepatol 2012;9:152-61.

9 Chiesa C, Andreoli GM, Pacifico L. Pediatric nonalcoholic fatty liver disease. J Pediatr 2019;95:4-6.

10 Doycheva I, Watt KD, Alkhouri N. Nonalcoholic fatty liver disease in adolescents and young adults: the next frontier in the epidemic. Hepatology 2017;65:2100-9.

11 Hagström H, Tynelius P, Rasmussen F. High BMI in late adolescence predicts future severe liver disease and hepatocellular carcinoma: a national, population-based cohort study in 1.2 million men. Gut 2018;67:1536-42.

12 Zhu J, Gao Y, Sun D, et al. Serum folate and cobalamin levels and urinary dimethylarsinic acid in US children and adults. Environ Sci Pollut Res Int 2018;25:17168-75.

13 GBD 2017 Disease and Injury Incidence and Prevalence Collaborators. Global, regional, and national incidence, prevalence, and years lived with disability for 354 diseases and injuries for 195 countries and territories, 1990-2017: a systematic analysis for the global burden of disease study 2017. Lancet 2018;392:1789-858.

14 GBD 2015 Mortality and Causes of Death Collaborators. Global, regional, and national life expectancy, all-cause mortality, and causespecific mortality for 249 causes of death, 1980-2015: a systematic analysis for the global burden of disease study 2015. Lancet 2016;388:1459-544.

15 Younossi ZM, Stepanova M, Rafiq N, et al. Nonalcoholic steatofibrosis independently predicts mortality in nonalcoholic fatty liver disease. Hepatol Commun 2017:1:421-8.

16 Ogden CL, Carroll MD, Lawman HG, et al. Trends in obesity prevalence among children and adolescents in the United States, 1988-1994 through 2013-2014. JAMA 2016;315:2292-9.
17 Flegal KM, Kruszon-Moran D, Carroll MD, et al. Trends in obesity among adults in the United States, 2005 to 2014. JAMA 2016;315:2284-91.

18 Ayonrinde OT, Oddy WH, Adams LA, et al. Infant nutrition and maternal obesity influence the risk of non-alcoholic fatty liver disease in adolescents. J Hepatol 2017;67:568-76.

19 Mor A, Svensson E, Rungby J, et al. Modifiable clinical and lifestyle factors are associated with elevated alanine aminotransferase levels in newly diagnosed type 2 diabetes patients: results from the nationwide DD2 study. Diabetes Metab Res Rev 2014;30:707-15.

20 Pedrelli P, Shapero B, Archibald A, et al. Alcohol use and depression during adolescence and young adulthood: a summary and interpretation of mixed findings. Curr Addict Rep 2016;3:91-7.

21 Oddy WH, Herbison CE, Jacoby $\mathrm{P}$, et al. The Western dietary pattern is prospectively associated with nonalcoholic fatty liver disease in adolescence. Am J Gastroenterol 2013;108:778-85.

22 Nanney MS, Lytle LA, Farbakhsh K, et al. Weight and weightrelated behaviors among 2-year college students. J Am Coll Health 2015;63:221-9.

23 Trépo E, Nahon P, Bontempi G, et al. Association between the PNPLA3 (rs738409 C>G) variant and hepatocellular carcinoma: Evidence from a meta-analysis of individual participant data. Hepatology 2014;59:2170-7.

24 Mencin AA, Loomba R, Lavine JE. Caring for children with NAFLD and navigating their care into adulthood. Nat Rev Gastroenterol Hepatol 2015;12:617-28.

25 van der Poorten D, Milner K-L, Hui J, et al. Visceral fat: a key mediator of steatohepatitis in metabolic liver disease. Hepatology 2008;48:449-57.

26 Mrad RA, Merjaneh N, Mubarak G, et al. The increasing burden of nonalcoholic fatty liver disease among young adults in the United States: a growing epidemic. Hepatology 2016;64:1386-7.

27 Mouzaki M, Trout AT. Virtual reality: new insights regarding the prevalence of nonalcoholic fatty liver disease in children and adolescents with obesity using magnetic resonance imaging. $J$ Pediatr 2019;207:8-10.

28 Hannon TS, Janosky J, Arslanian SA. Longitudinal study of physiologic insulin resistance and metabolic changes of puberty. Pediatr Res 2006;60:759-63.

29 Chalasani N, Younossi Z, Lavine JE, et al. The diagnosis and management of nonalcoholic fatty liver disease: practice guidance from the American association for the study of liver diseases. Hepatology 2018;67:328-57.

30 Golabi P, Otgonsuren M, de Avila L, et al. Components of metabolic syndrome increase the risk of mortality in nonalcoholic fatty liver disease (NAFLD). Medicine 2018;97:e0214

31 Hossain N, Afendy A, Stepanova M, et al. Independent predictors of fibrosis in patients with nonalcoholic fatty liver disease. Clin Gastroenterol Hepatol 2009;7:1224-9.

32 Vos MB, Abrams SH, Barlow SE, et al. NASPGHAN clinical practice guideline for the diagnosis and treatment of nonalcoholic fatty liver disease in children: recommendations from the expert Committee on NAFLD (ECON) and the North American Society of pediatric gastroenterology, hepatology and nutrition (NASPGHAN). J Pediatr Gastr Nutr 2017;64:319-34. 